\title{
What do we know? What do we need to know?
}

\author{
Milena J. Henzlova, $M D{ }^{a}$ and W. Lane Duvall, $M D^{b}$ \\ a Division of Cardiology, Mount Sinai Medical Center, New York, NY \\ b Division of Cardiology, Hartford Hospital, Hartford, CT
}

Received Jul 29, 2016; accepted Jul 29, 2016

doi:10.1007/s12350-016-0640-y

\section{See related article, pp. 245-251}

Nuclear cardiology experienced unprecedented growth in the 1980-1990's after the introduction of SPECT technology, development of Tc-99mbased tracers, ${ }^{1}$ and utilization of coronary vasodilators for pharmacologic stress. ${ }^{2-4}$ During the late part of the last century, nuclear cardiology became a dominant physiologic and noninvasive method for the diagnosis, risk stratification, and prognostication of epicardial coronary artery disease (CAD). However, the arrival of competing diagnostic modalities used for similar indications, such as coronary CT angiography and stress echocardiography, unmasked weaknesses of the radionuclide methods, particularly the length of the procedure, relatively high radiation doses for a diagnostic test,${ }^{5,6}$ and possible underestimation of the extent of the disease. ${ }^{7}$

Introduction of solid-state cameras almost 10 years ago handed a needed lifeline to the stagnant technology. Increased imaging efficiency promised to accomplish both goals: shorter imaging times and lower radiation doses. Two systems equipped with Cadmium-Zinc-Telluride (CZT) detectors became commercially available: the Discovery 530 (GE Healthcare) and the D-SPECT (Spectrum Dynamics) cameras. ${ }^{8,9}$

Early adopters of the high-efficiency cameras experimented with optimal Tl-201 and Tc- $99 \mathrm{~m}$ doses, imaging times, and technicalities of image acquisition. The first papers appeared in 2009-2010 and explored image quality, diagnostic utility in a variety of patient

Reprint requests: W. Lane Duvall, MD, Division of Cardiology,

Hartford Hospital, Hartford, CT; Lane.Duvall@hhchealth.org

J Nucl Cardiol 2017;24:252-4.

1071-3581/\$34.00

Copyright (C) 2016 American Society of Nuclear Cardiology. populations, and compared images acquired on traditional Na-I cameras with the new technology..$^{10,11}$

The ability to risk stratify and prognosticate future events is a cherished strength of SPECT imaging. ${ }^{12} \mathrm{We}$ now have data from 6 nuclear cardiology laboratories documenting the prognostic value of CZT SPECT imaging using lower radiation doses and shorter imaging times.

The latest contribution to the data is a paper from Sao Paolo, Brazil. ${ }^{13}$ The authors followed two cohorts of SPECT MPI patients for 34 and 33 months, respectively, prior to and after the acquisition of CZT technology. Group $1 \quad(\mathrm{~N}=1,777$ patients $)$ underwent two-day (stress-rest) Tc-99m SPECT myocardial perfusion imaging (MPI) using a traditional Na-I camera during the years 2008-2010. Group $2(\mathrm{~N}=1,777$ patients) underwent one-day (rest-stress) Tc-99m SPECT MPI using a CZT camera during the years 2011-2012. The patients were recruited from an initial pool of 6,128 patients, and propensity score matching was employed to make comparison possible. Group 1 (Na-I) had a slightly higher number of abnormal scans $(27.4 \%$ vs $21.6 \%, p<0.001$ ) compared to Group 2 (CZT) despite the demographic and risk factor matching used. The number of hard events in patients with a normal study was also higher in Group 1 (1.0\% per year) compared to Group $2(0.5 \%$ per year $)(p<0.001)$. However, there was no difference in the number of hard events and revascularizations in patients with abnormal perfusion between the two groups during the follow-up $3.3 \%$ per year vs $3.2 \%$ per year, and $6.6 \%$ per year vs $6.3 \%$ per year, respectively). The current study therefore confirms the noninferiority for medium-term (33-34 months) prognosis of MPI results obtained from low-dose highefficiency SPECT studies compared to the results obtained from standard-dose Na-I SPECT imaging. This is despite the shorter acquisition time and low administered activity with the high-efficiency camera. The imaging time for the Na-I camera (two-day test) was 15 minutes, compared to 10 minutes using the CZT camera (one-day test), and the average Tc- $99 \mathrm{~m}$ dose was 
25-40 $\mathrm{mCi}$ for the Na-I camera and $20 \mathrm{mCi}$ for the $\mathrm{CZT}$ camera.

There are a number of weaknesses of the presented data. First, the non-simultaneous inclusion of the patients and temporal separation of the groups (before and after adoption of new technology) is not ideal, although this is somewhat offset by the use of propensity matching. Second, there is a disparity in the use of imaging protocols. A stress-rest two-day test was used for the traditional Na-I camera and a one-day rest-stress test employed for the CZT cohort. Therefore, the comparison between groups includes not only camera technology, image acquisition time, and administered activity, but also imaging sequence and timing. Neither of these first two factors allows for a meaningful explanation of the disparity in number of abnormal scans and less benign prognosis of a normal scan with the Na-I camera. Finally, the study lacks analysis and comparison of prognosis based on the perfusion defect size (mild, moderate, severe) which is a cornerstone of myocardial perfusion imaging.

Nevertheless, the authors' conclusions are consistent with previous studies utilizing high-efficiency SPECT cameras. Duvall et al followed 131 patients imaged with a high-efficiency camera with reduced acquisition times and $1 / 2$ of the traditional administered activity for one year. ${ }^{14}$ Patients with normal perfusion had a benign prognosis with no cardiac deaths or nonfatal MI, and $2.3 \%$ of patients undergoing percutaneous coronary intervention. Nakazato et al followed 1,613 consecutive patients imaged using a solid-state camera for 2.6 years. ${ }^{15}$ Prognosis was similar to results obtained using Na-I camera and was dependent on the perfusion defect size. The annual major adverse cardiac event (MACE) rate of a normal scan was $0.87 \%$. Chowdhury et al followed 1,109 consecutive patients undergoing a two-day stress-rest study imaged with a CZT camera for 624 days (approximately 21 months). ${ }^{16}$ The MACE rate was $0.4 \%$ for normal scans and $6.8 \%$ for abnormal scans. The results with the high-efficiency camera (imaging time 3-10 minutes, $15 \mathrm{mCi}$ Tc-99m tetrofosmin for both stress and rest imaging) were consistent with imaging using a traditional SPECT camera. Oldan et al followed 2,088 patients for 2 years who underwent a two-day stress-rest SPECT during the same time period and were imaged using either a Na-I camera $(\mathrm{N}=1,128$ patients) or a CZT camera $(\mathrm{N}=865$ patients). ${ }^{17}$ The number of hard events was similar using both technologies. Einstein et al followed 69 patients with a normal stress-only SPECT referred from the Emergency Department for the evaluation of acute chest pain. ${ }^{18}$ Imaging was done on a CZT camera with Tc$99 \mathrm{~m}$ tetrofosmin with very low radiation exposure to the patient $(0.99 \mathrm{mSv})$. There were no major adverse cardiac events at one year of follow-up. Yokota et al followed 1,650 consecutive (nonparallel) patients for 37 months with normal stress-first, CT attenuation corrected SPECT: 362 patients were imaged using a Na-I camera, and 1,288 patients were imaged using a CZT camera. ${ }^{19}$ Stress-only imaging was used in $37 \%$ of patients imaged with the Na-I camera and in $61 \%$ of patients imaged with the CZT camera. The annual MACE rate was $2 \%$ and $1.5 \%$, respectively ( $p=0.08)$.

Even with different protocols, follow-up times and population cohorts, the message is similar: in more than 10,000 patients, the prognostic value of a normal and an abnormal stress SPECT study is similar if imaging is done using a traditional Na-I camera or a high-efficiency camera. However, imaging times are shorter and radiation doses are lower using the new technology.

What else has been learned in the past 7 years from the early adopters of solid-state cameras?

(1) Radiation doses can be markedly decreased for both Tc-99m and Tl-201.

(2) Imaging times are decreased. Further lowering of radiation doses can be exchanged for longer imaging time.

(3) Normal SPECT (rest-stress, stress-rest, or stressonly) and abnormal SPECT have similar risk stratification and prognostication value using both types of cameras.

(4) Correlation with angiographic results is acceptable and comparable to imaging with Na-I cameras.

(5) Obesity is a challenge despite of the optimistic conclusions of some papers. ${ }^{20,21}$ Most studies with CZT cameras excluded obese and morbidly obese patients. The literature suggests that perhaps DSPECT camera (Spectrum Dynamics) is more suitable for imaging obese patients compared to the Discovery 530 (GE Healthcare). ${ }^{22}$

What do we need to know in the future? Only preliminary data are available for some of the following:

(1) Is simultaneous Tl-201/Tc-99m dual-isotope imaging with lower doses of both tracers, and Tl-201/I123 MIBG imaging feasible? ${ }^{23}$

(2) Is dynamic acquisition with possible coronary flow reserve and/or coronary flow determination possible ${ }^{24}$

(3) Will there be a role for CZT cameras for gated blood pool imaging with reduced radiation dose in oncology patients? ${ }^{25}$

(4) Attenuation correction remains a challenge. Most users include prone in addition to supine imaging (made easy with short imaging time) in their standard imaging protocol. The addition of affordable CT attenuation with a coronary calcium score evaluation 
option would enormously improve diagnostic confidence, risk stratification, prognostication, and therapeutic decision making.

(5) Future clinical trials utilizing SPECT imaging should include solid-state cameras for time to diagnosis, radiation dose, and cost effectiveness.

\section{References}

1. Holman BL, Jones AG, Lister-James J, Davison A, Abrams MJ, Kirshenbaum JM, et al. A new Tc-99m-labeled myocardial imaging agent, hexakis(t-butylisonitrile)-technetium(I) [Tc-99m TBI]: initial experience in the human. J Nucl Med 1984;25: 1350-5.

2. Leppo JA. Dipyridamole myocardial perfusion imaging. J Nucl Med 1994;35:730-3.

3. Cerqueira MD, Verani MS, Schwaiger M, Heo J, Iskandrian AS. Safety profile of adenosine stress perfusion imaging: Results from the Adenoscan Multicenter Trial Registry. J Am Coll Cardiol 1994;23:384-9.

4. Iskandrian AE, Bateman TM, Belardinelli L, Blackburn B, Cerqueira MD, Hendel RC, et al. Adenosine versus regadenoson comparative evaluation in myocardial perfusion imaging: Results of the ADVANCE phase 3 multicenter international trial. J Nucl Cardiol 2007;14:645-58.

5. Einstein AJ, Moser KW, Thompson RC, Cerqueira MD, Henzlova MJ. Radiation dose to patients from cardiac diagnostic imaging. Circulation 2007;116:1290-305.

6. Einstein AJ, Henzlova MJ, Rajagopalan S. Estimating risk of cancer associated with radiation exposure from 64-slice computed tomography coronary angiography. JAMA 2007;298:317-23.

7. Gimelli A, Liga R, Duce V, Kusch A, Clemente A, Marzullo P. Accuracy of myocardial perfusion imaging in detecting multivessel coronary artery disease: A cardiac CZT study. J Nucl Cardiol 2016. doi:10.1007/s12350-015-0360-8.

8. Slomka PJ, Dey D, Duvall WL, Henzlova MJ, Berman DS, Germano G. Advances in nuclear cardiac instrumentation with a view towards reduced radiation exposure. Curr Cardiol Rep 2012; 14:208-16.

9. Buechel RR, Gaemperli O. Newer generation cameras are preferred. J Nucl Cardiol 2016;23:790-4.

10. Gambhir SS, Berman DS, Ziffer J, Nagler M, Sandler M, Patton J, et al. A novel high-sensitivity rapid-acquisition single-photon cardiac imaging camera. J Nucl Med 2009;50:635-43.

11. Esteves FP, Raggi P, Folks RD, Keidar Z, Askew JW, Rispler S, et al. Novel solid-state-detector dedicated cardiac camera for fast myocardial perfusion imaging: Multicenter comparison with standard dual detector cameras. J Nucl Cardiol 2009;16:927-34.

12. Hachamovitch R, Berman DS, Kiat H, Cohen I, Cabico JA, Friedman $\mathrm{J}$, et al. Exercise myocardial perfusion SPECT in patients without known coronary artery disease: Incremental prognostic value and use in risk stratification. Circulation 1996;93:905-14.

13. Lima R, Peclat T, Soares T, Ferreira C, Souza, AC, Camargo G. Comparison of the prognostic value of myocardial perfusion imaging using a CZT-SPECT camera with a conventional anger camera. J Nucl Cardiol 2016. doi:10.1007/s12350-016-0618-9.

14. Duvall WL, Croft LB, Ginsberg ES, Einstein AJ, Guma KA, George $\mathrm{T}$, et al. Reduced isotope dose and imaging time with a high-efficiency CZT SPECT camera. J Nucl Cardiol 2011;18: 847-57.

15. Nakazato R, Berman DS, Gransar H, Hyun M, Miranda-Peats R, Kite FC, et al. Prognostic value of quantitative high-speed myocardial perfusion imaging. J Nucl Cardiol 2012;19:1113-23.

16. Chowdhury FU, Vaidyanathan S, Bould M, Marsh J, Trickett C, Dodds $\mathrm{K}$, et al. Rapid-acquisition myocardial perfusion scintigraphy (MPS) on a novel gamma camera using multipinhole collimation and miniaturized cadmium-zinc-telluride (CZT) detectors: Prognostic value and diagnostic accuracy in a 'realworld' nuclear cardiology service. Eur Heart J Cardiovasc Imaging 2014; 15:275-83.

17. Oldan JD, Shaw LK, Hofmann P, Phelan M, Nelson J, Pagnanelli $\mathrm{R}$, et al. Prognostic value of the cadmium-zinc-telluride camera: A comparison with a conventional (Anger) camera. J Nucl Cardiol 2015. doi:10.1007/s12350-015-0181-9.

18. Einstein AJ, Johnson LL, DeLuca AJ, Kontak AC, Groves DW, Stant J, et al. Radiation dose and prognosis of ultra-low-dose stress-first myocardial perfusion SPECT in patients with chest pain using a high-efficiency camera. J Nucl Med 2015;56:545-51.

19. Yokota S, Mouden M, Ottervanger JP, Engbers E, Knollema S, Timmer JR, et al. Prognostic value of normal stress-only myocardial perfusion imaging: a comparison between conventional and CZT-based SPECT. Eur J Nucl Med Mol Imaging 2016;43:296-301

20. Gimelli A, Bottai M, Giorgetti A, Genovesi D, Filidei E, Marzullo P. Evaluation of ischaemia in obese patients: feasibility and accuracy of a low-dose protocol with a cadmium-zinc telluride camera. Eur J Nucl Med Mol Imaging 2012;39:1254-61.

21. De Lorenzo A, Peclat T, Amaral AC, Lima RS. Prognostic evaluation in obese patients using a dedicated multipinhole cadmiumzinc telluride SPECT camera. Int J Cardiovasc Imaging 2016;32: 355-61.

22. Nakazato R, Slomka PJ, Fish M, Schwartz RG, Hayes SW, Thomson LE, et al. Quantitative high-efficiency cadmium-zinctelluride SPECT with dedicated parallel-hole collimation system in obese patients: results of a multi-center study. J Nucl Cardiol 2015;22:266-75.

23. Kobayashi M, Matsunari I, Nishi K, Mizutani A, Miyazaki Y, Ogai K, et al. Simultaneous acquisition of (99m)Tc- and (123)Ilabeled radiotracers using a preclinical SPECT scanner with CZT detectors. Ann Nucl Med 2016;30:263-71.

24. Nkoulou R, Fuchs TA, Pazhenkottil AP, Kuest SM, Ghadri JR, Stehli J, et al. Absolute Myocardial Blood Flow and Flow Reserve Assessed by Gated SPECT with Cadmium-Zinc-Telluride Detectors Using 99mTc-Tetrofosmin: Head to Head Comparison with 13N-Ammonia PET. J Nucl Med 2016. doi:10.2967/jnumed. 115.165498.

25. Jensen MM, Schmidt U, Huang C, Zerahn B. Gated tomographic radionuclide angiography using cadmium-zinc-telluride detector gamma camera; comparison to traditional gamma cameras. J Nucl Cardiol 2014;21:384-96. 